\title{
An Uncertain Future: The Unchanging Views of Care Home Residents about Living and Dying
}

\section{ABSTRACT}

Older people living in a care home have a limited life expectancy and care homes are an important setting for end of life care provision. This research aimed to explore the views, experiences and expectations of end of life care among care home residents to understand if key events or living in a residential environment influenced their views. The research used a prospective design. The paper draws on the data of 121 care home residents (a sub-sample of 63 were interviewed three times over a year) recruited from six care homes (without on-site nursing) in the UK. Four main themes were identified; Living in the Past, Living in the Present, Thinking about the Future and Actively Engaged with planning the future. Many residents said they had not spoken to the care staff about end of life care; many assumed their family or General Practitioner would take responsibility. Core to the older person's ability to discuss end of life care is their acceptance of being in a care home, the involvement of family members in making decisions and the extent to which they believed they could influence decision making within their everyday lives. 


\section{Introduction}

Care homes for older adults are an important setting for end of life care provision. In the UK there are an estimated 15,700 care homes providing nursing care and/or personal care to more than 400,000 older people with a range of different needs in England and Wales. In 2008, $0.8 \%$ of $65-74$ year olds lived in a care home or long stay hospital setting which rose to $16.2 \%$ for people aged 85 and over ${ }^{1}$. Approximately $21 \%$ of people over the age of 65 die in care homes ${ }^{2}$. Dying is not an unexpected outcome following a move into a care home, although often not acknowledged at the time of entry. Staff working in care homes have a potentially difficult role when looking after residents who are living and residents who are dying both alongside each other ${ }^{3}$. This paper describes how older people experience living in a care home and how, over time this influences their anticipation and planning for the end of their lives.

\section{Background}

Only a small percentage of residents in care homes die from malignant disease; those who are over 85 years, living with dementia and or multiple health conditions may follow much more unpredictable pathways to death ${ }^{4,5}$. Dying is part of care home life and an important aspect of the overall quality of care provided. In England, despite recent initiatives to improve this populations's access to palliative care services ${ }^{6,7}$ the experience of the older person is largely unknown. Studies often rely on proxy accounts and retrospective data that focuses on prognostication or the management of symptoms ${ }^{8,9,10}$. How individuals engage with the anticipation of dying and if living in a care home affects their decisions and need for support is unknown ${ }^{11}$. It is also only partially understood how the culture and organisation of a home affects end of life care or if different dying trajectories, high levels of cognitive impairment and co-morbidity affects the recognition of need and access to services. 
This study aimed to understand how living in a care home influences older people's views, experiences and expectations of end of life care and symptom relief. To the best of our knowledge, this is the first English study to interview care home residents at different time intervals over a year and examine how their views and expectations are shaped by the experience of living in care homes.

\section{Method}

The study used a prospective design with a mixed method approach. Six care homes (without onsite nursing) in three disparate geographical areas were purposively selected to include a range of providers (not for profit, faith based, large chain), different in size and variable staff training on end of life care.

The care homes were identified using the Commission for Social Care Inspection (CSCl) Directory (now Care Quality Commission). Selection and inclusion criteria for care homes were; providing care for older people, no on-site nursing, size 27-60 places and a favourable CSCI inspection report (to exclude homes that might be subject to special measures or in danger of closing). Care home managers were invited to participate in the study by letter and those who expressed an interest were then visited by the researchers. Once the manager had given consent, the researchers provided potential participants (residents, care home staff and health professionals) with information sheets about the study.

The research aimed to be as inclusive as possible, inviting all residents to take part in the study, including residents living in any dementia units ${ }^{12,13}$. It was an assumption of the researchers that many older people and those with dementia would wish to be listened to and influence practice ${ }^{14,15}$ Recruitment involved several approaches: including letters of invitation with information leaflets to residents, posters with photographs of the researchers placed on care home notice boards 
publicising the study, coffee mornings with residents and individual discussions with older people about what participation would involve. Four lay members of the University of X Public Involvement in Research (PIR) who had experience of relatives and friends living in care homes assisted the study. They were of a similar age to some of residents and were able to answer questions and discuss the study independently of the research team at the coffee mornings and during further visits. All residents who had capacity to understand the purpose of the study and consented to participation had their care notes reviewed four times over a year and a self-selected sub-sample agreed to be interviewed three times. Consent to interview was an ongoing process throughout the period of data collection period $^{16,17,18}$

The interviews enabled residents to talk about what was significant to them about living and dying in a care home. Prompts were used to ask about their background, their health, life in the care home, health services received, the context and process of care, and their thoughts about their future and about death. The interviews were digitally recorded and later transcribed verbatim and anonymised. The care notes were reviewed for background information (diagnosis, medication), review of NHS input, assessments, change in care, health status and any records of end of life plans or wishes.

Data analysis of the interviews used the computer package NVIVO to map the data. The analysis involved three stages. First, a process of familiarisation and decontextualisation and segmenting of data into separate and defined categories that were close to the participants' own categories. Second, comparison was made within and between categories which enabled the identification of preoccupations, differences and themes. The third stage was the identification of relationships and working hypotheses. Credibility of analysis was sought through searching for rival explanations, peer debriefing within the research team, and discussion with the PIR group. In addition, the PIR members tested the validity of the findings at the end of the study by running three discussion groups with the care home residents. 
Ethical review and health and social care governance permissions were secured through the NHS NRES (08/H0502/38) and local government and NHS organisations.

\section{Findings}

The six care homes varied in their characteristics. There was a range of occupancy (27-60), size and religious affiliation. In terms of approaches to end of life; one care home had formal end of life procedures and another had its own final wishes forms. The other care homes all had funeral arrangement forms and reported that they dealt with end of life decisions "as and when".

Of the 257 older people who were resident in the care homes at the start of the study, 121 agreed to participate in the study (47.1\%) and their case notes were reviewed. Of these 121 residents, 63 from across the six care homes were interviewed up to 3 times over the period of the study; 23 died during this period. The characteristics of the sample are given in Table 1 and were broadly representative of the wider care home population.

\section{Table 1 here}

Nearly half (46.2\%) the residents had 3 or more co-morbidities recorded (median 2, mean 2.7). The three most common recorded diagnoses were dementia (38\%), disorders of the circulatory system (excluding CVA) (38\%) and musculoskeletal system (28\%). The overall Barthel scores are shown in Table 2. A significant difference was found in the mean Barthel scores of the homes (one way ANOVA, $\mathrm{p}<0.05$ ). Dependency was lowest in home 1 (mean Barthel score 14.2) and highest in home 3 (mean Barthel score 9.6).

\section{Table 2 here}

It is a limitation of the Barthel score that it does not account for cognitive impairment. 
The findings presented here refer to the 63 older people who were each interviewed three times over the year of data collection. The study assumed that living in a care home, observing other residents dying and experiencing episodes of ill health would, over time, shape how residents talked about their own mortality and their priorities for end of life care. The findings did not support this. Although there was a wide variation in response (figure 1), very few participants changed their views or priorities over the year of data collection. Nor did the culture as measured by a tool to establish the attitudes and belief systems of the care homes ${ }^{19}$ and espoused approach to end of life care of the six care homes appear to influence participants' responses. Preoccupations and priorities for end of life care reflected four main themes (and subgroups) which are detailed in figure 1: Living in the Past, Living in the Present, Thinking about the Future and Actively Engaged with planning the future. A minority were able to anticipate their death and were able to discuss their plans and wishes. Importantly, residents did not necessarily hold one view consistently; even within the same interview they moved between these overlapping frameworks.

\section{Figure 1 here}

\section{Living in the past}

Among those residents who were not reconciled to living in a care home, discussing their future focused on either returning home, or moving in with relatives, or their sadness at no longer being able to cope with living independently at home. Typically these respondents said "I want to go home". One resident repeated this wish to go home in the interviews held across the 12 month data collection period. Questions about the future and planning for end of life in the care home seemed irrelevant to these residents. For example, one said:

"I'm going to see what happens when I get home" (3-002-1)( $1^{\text {st }}$ interview) 
Although their plan to move back home (often by their own admission) might be unrealistic, this 'hope' (their connection with the past) appeared to be a means of coping with their situation. For this group of residents their future lay back in the past.

Living in the past could also reflect the person's cognitive state at the time of the interview, when talking about the future could be inappropriate if it was contradicting the person's sense of reality. Planning for end of life care with those residents meant conversations had to be tailored very differently. People living with dementia or "short term memory problems" could talk about dying and what had been good and bad in the deaths of loved ones and what was important to them now (for example, security and comfort):

"I mean, I know [husband] is dead, but he had cancer so I didn't want him to go on living" (1006-1)

The interviews also demonstrated that over time opportunities to talk about living and dying could decrease. One male resident aged over 90 moved to a dementia unit of the care home during the study. In the first interview he had been oriented and knew his age and why he was in a care home but, by the third interview he said he was looking forward to retirement, being 'demobbed' (moving back to civilian state from active armed service) and spending time in the garden; thus the opportunity to talk about the future, and about his views and priorities had by this third interview changed:

"So I'm now looking forward to retirement from [work place] and my old age pension and a bit of savings, I shall be all right" (1-005-3)

\section{Living in the present}

Across the six care homes many of the older people did not think they could plan for the future. For them the future was by definition uncertain and they were unconvinced that planning ahead would be helpful. Their experiences prior to entering the care home had taught them that; 
“(Laughs) It's very difficult to plan isn't it? You don't know how long... you don't know how far away your future is do you and how far... how long I'm going to live for" (2-017-1)

"Yes. Its there, something [dying] that happens to all of us sometime or other, whether it's easy or not depends upon the time. I mean, we can't plan it" (6-007-3)

“Well I can't have plans can I because this is my final home I think, and I'm 81 , I hope I might have another at least ten years of living here, you know, and I don't know, I just, I try not to project too much, you know, as to what could happen" (1-013-1)

For these older people there were no more plans to be made; death was an inevitability, and they had no control over when this might happen. The majority of older people who focused on the present described themselves as living day-to-day. These views tended to be consistent over time. The following quotes provide examples of their reasoning and demonstrate how they did not change over time:

"Well you sort of jog along, you know take each day as it comes" (2-017-2)

“Well I don't know about the future, I just go along day by day. I don't say l'd recommend it, because nobody wants to be here, but I haven't got anything to say against it. If people say to me are you happy here, I say I am, I am happy" (2-005-1)(1 $1^{\text {st }}$ interview)......

...."Nothing really, it's just, it's really, it's just an existence, you live from one day to another, it's all the same, $\mathrm{mmm}$ " $(2-005-3)\left(3^{\text {rd }}\right.$ interview $)$.

"No, it's never occurred to me really [to think about death]. Rather let nature take its course" (1-017-3)

There were some reference to fears about the future "probably hit me badly" (1-09-3) but their stated preference was not to think about it. The fact that they may not have chosen to live in a care 
home, but accepted it was necessary, underlined the feeling that they had limited control over their current experience and therefore found it difficult to envisage control over the future.

\section{Thinking about the future}

In contrast, a group of older people, though not preoccupied about what might happen in the future, were more able and open to discuss future plans and preferences for end of life care. These residents accepted that they would be in the care home until they died; "the future? Just to die I think" (1-004-2). This group used words such as 'resigned', 'prepared', 'quite settled';

“I'm quite happy here as I say, yes. I'm quite... I'd quite like to live here for the rest of my life as long as I can" (1-002-3)

Older people did not see that their wants or preferences were that important. Concerns about family members and their wellbeing were more likely to feature as key issues when discussing priorities and preferences for end of life:

"I think that (planning for the future) might make quite a difference to them [relatives], because I mean I'm quite happy, I accept the fact that one day I'm going to pop off, and it doesn't worry me in the least. I just know that I'm going because you've got to, you'd get in the way otherwise [laughs]. So, you know, there's no great query in my life that there should be anything to worry about, because I don't really worry about it. If I pop off, so be it. Which doesn't really help much, well I don't know, perhaps it does, it saves people worrying about me worrying" (5-004-1)

Residents who had talked with family members about death did not have strong opinions on wanting them with them when they were dying. They said "it's up to them" and felt that their 
sons/daughters might not cope very well. There did not seem to be much concern among those interviewed about dying alone.

Few older people $(n=6)$ talked explicitly about their age and limited life expectancy. They felt they had achieved 'a good age' and acknowledging that as their children were 'sorted now' they didn't have to worry about them anymore. It was as if they were describing the natural order ("allotted time"), and looking back on their life, they could not influence things anymore:

“Well my future is very limited you know, I'm 96 [participant's emphasis] now" (1-001-1)

This view of a limited future following a long life, contrasted with those residents who felt that there was no future left now they were in the care home. This group of residents were sad and depressed about their lives in the care home. There were examples of this in all six care homes. Loss of purpose and the limited ability to make a contribution were recurrent themes in some participants' discussion about the future and its pointlessness. This quote below demonstrates both the impact of the feeling of having no purpose and that care home staff may not have acknowledged the significance of these feelings:

"I should love to die. I don't want to go on existing at all. It seems to me quite non-sensible to go on existing as I am. I don't go on letting this out, I don't talk about it. I don't suppose I'm the only one either"

Interviewer: Have you talked to anybody about it?

“Yes I told (care home manager) I've forgotten what brought it up because I wasn't going to tell her but I did and she said "oh, you've got lots of people to take an interest in" (6-008-1)

\section{Actively engaged with planning for the future}


A few of the older people $(n=7)$ interviewed spoke of active plans for the future. What they wanted to happen in the period before death (as opposed to after death - funeral, burial arrangements). Some previous professional health knowledge, recent hospital experience, experience of death and religious views appeared to be influential in helping them to articulate what they might want to happen at the end of their own life.

Residents with Professional Health Knowledge: Two residents, both former health professionals, were the most articulate in discussing their advance care plans. They were very clear that they did not want resuscitation, invasive treatment and any unnecessary hospital admissions. They were very specific with plans including details of not wanting major operations, long periods of hospitalisation (unless to make more comfortable), did not want to be kept artificially alive (no more than one month), one saying the essence of her plan was:

“That I don't want to be resuscitated, I want to be let alone to die normally, that's the biggest thing" (3-001-1)

During the study, this resident was admitted and returned from hospital and later died receiving support in the care home and surrounded by her family.

The second participants' views and plans for the future became more defined throughout the 12 months of the research and were actively facilitated by the care home manager. This was the only example of where decision-making and the development of a plan involved a member of staff. At the initial interview the resident had been left with some forms to fill in; by the second interview she had had a discussion with the manager

“if I have a fall and I might be injured I don't mind going to hospital but I don't want to be kept in unless I've broken something and if they decide I'm dehydrated, as I read about a case, I said I don't want to be kept in, I'd rather come back here and be dehydrated, than in hospital. 
Well I said that I don't want to be... If I die I don't want to be resuscitated, if you know what I mean" (4-001-2) (2 $2^{\text {nd }}$ Interview)

By interview three, she had written down her wishes;

Residents with recent hospital experiences: While many residents were ill, this did not seem to increase any pre-occupation with death. Their views were consistent throughout the study even though some experienced illness or an incident (e.g. an acute infection, a fall, a hospitalisation) and/or were aware of other residents dying. Three older people were more articulate than others about their future wishes after a return from hospital. They were clear they did not to be admitted to hospital again. It was unclear as to how, or if, these views had been recorded in the home's care notes. Their wishes stemmed from negative feelings about their hospital experience and their opinion that there was little benefit to them from hospital attempts to prolong life.

Religion/Faith: The religious background of the residents also affected their acceptance of the future and of death. Two of the care homes had a faith base and these homes provided structure and reasons to acknowledge death (visiting residents in their rooms to pray, a religious minister visiting, music played when the deceased resident left the home). On a personal basis religion provided individuals with a great comfort, reassurance and a belief or certainty as to what would happen in the future. However there was no evidence of more advance care plans in these homes than in other homes.

\section{Preferred Place of Care - Care Home or Hospital?}

The majority of residents, when asked if they would like to stay in the care home or go to hospital at the end of their life, said they would choose to stay where they were. This may be a positive choice for some residents, however for others it was a desire not to move (again) and this meant the care home by default: 
“I'm satisfied, I don't want to move, I just want to stay here until I die, that's all I want. I want to stay here until I die" (3-003-1)

The reasons they offered were that they valued the presence of familiar staff, had a dislike of hospitals and a positive opinion of the care home "if you can't be at home you couldn't be in a better place" (3-014-3). Despite the majority of residents wanting and assuming they would be able to stay in the care home, few had been asked to sign anything to ensure that this would happen and little evidence of their wishes was apparent in the care notes. Residents who said they would like to stay in the care home also knew that they might not be able to stay. If they became very ill they might have to move to a nursing home or a hospital and they felt this would not be their decision, and it would probably be made by a General Practitioner.

This further feeling of uncertainty, of another possible move, equated illness with the likelihood of needing specialist nursing help (i.e. from hospital nurses, outside capabilities of the care home staff) rather than needing less intervention and palliative care being brought into the care home. Many residents felt they were not able to exercise choice:

\footnotetext{
"Yes, I seem to have that [what will be will be] sort of firmly fixed in my mind but I mean I can't do anything so really I just have to cope with whatever crops up, there's no choice is there" (5-004-3)
}

There was a small number of other residents $(n=7)$ who appeared not to mind whether they remained in the care home or went to hospital. These felt they would be unaware of what was happening at the time. A very small minority $(n=3)$ said they would prefer to go to hospital to die ( $n=17$ did not answer this question directly).

\section{Choice at End of Life}

The choices for end of life care are not straight forward for people living in a care home. Firstly, many of these residents are not identified as being, or do not see themselves as being at the 'end of 
life'. Secondly, many residents were not very concerned about or aware of end of life care choices. Thirdly, the choices available are far more complex than a simple choice of either/or judgements. Some residents would prefer to move back to their own home again and die there or move in with relatives; however, such options were often not available. One resident admitted she had "given up responsibility" (1-06) when she came into the care home. Giving residents the choice of where they would like to die is for some a choice too late or lacking reality. It is possible that for some participants the process of giving up much of their independence meant they did not expect to participate in future care-related decisions or had made such choices. Many residents said they had talked to relatives and that [it] was all 'sorted out'. The residents did not often elaborate and these discussions and related decisions were not recorded. For others family members needed to be involved:

"I think they should say to the family of the person ...if they're extremely ill, have you got any feelings about it and have you ever talked to her or him about it?" (5-001-3)

Residents appeared to imply that these decisions may not be ones they were able to or wished to make on their own. Many felt it would be a medical decision, taken by the GP or by those in the care home.

Very few residents said they had had a conversation with the care home staff concerning end of life. They were asked if they would like to talk to staff about end of life, and the majority said they did not want to, even though for some the care homes were using care home specific palliative care support tools including advance care plans. The residents' impression was that the staff might not be that interested, that family members were the ones to talk to (rather than staff because "it's private") or that there was nothing to talk about.

\section{Conversations about living not dying}


The lack of conversations about end of life (and plans and records) in the care home revealed a lack of in-depth conversations between staff and residents. Residents wanted to have someone to talk to about their past, their life in the care home or just to be listened to. Residents described the staff as friendly, but felt they did not have the time to have the kind of conversation that could begin to talk about end of life. One finding of the study was that many participants commented on how helpful the interview process had been, possibly because the researcher gave participants time and met with them three times over a year to listen to their views and priorities:

"Interviewer: ..have you ever talked to any of the staff about your wishes for the end of your life?

No, I never said anything to anybody, you're the first one I've ever said anything" (2-012-1)

"I mean I can talk to you which is marvellous and there's not very many people I can talk to, I just have to...don't make a fuss about it because it isn't, it's just that my inside I'm not, I don't know how to put it but I just used to be a person who could do everything as required and now I don't, it's annoying" (5-009-3)

\section{Discussion}

The research revealed that older people are able to talk about living and dying, over time, but do not conceptualise these as areas where it may be possible to do so in terms of options and choices regarding end of life care. There was a wide range of opinion and differences in the amount of engagement with discussion about dying. A degree of acceptance of their situation in the care home seemed to have some bearing on residents' ability to plan for the future. Other studies with community dwelling older people have also acknowledged the diversity of views within this age group ${ }^{20,21}$. These choices are often not made autonomously by the older person; but are often made with or by other relatives on their behalf ${ }^{22,23,24}$. This emphasises the need for an 
understanding of older people's preferences for care, the influences of their personal biographies and their readiness to engage with conversations about dying.

Contrary to expectation, older people's views about dying did not change over time and were not dissimilar to the views of the wider population. Equivalent attitudes of "just live for today " have been found among older people living in the community ${ }^{20,25,24}$, in long-term care ${ }^{3}$ and even among young athletes ${ }^{26}$. A small minority of residents had made active plans for their end of life, seemingly influenced by their health professional knowledge, recent hospital experience, previous experience of death or religious beliefs. Particular experiences (hospital admissions, falls) may lead plans to change and may provide the opportunity to initiate (or review) discussions about any initial thoughts or amendments of end of life plans.

End of life care planning is a process of discussion and review ${ }^{27,28}$. This study has suggested the value of ongoing discussions with older care home residents that foster opportunities to talk about dying but recognise the importance of the involvement of families (if available and willing) and possibly care staff. It also suggests that providing opportunities to talk about the future as early as possible (for those with a diagnosis of dementia) may be important ${ }^{10 .}$ Although, solely enhancing older people's opportunities to be involved in decision making towards the end of life, does not guarantee of itself the quality of end of life care.

Studies that have sought to use advance care planning and other palliative care support tools to reduce hospital admissions from care homes have demonstrated that this may be possible ${ }^{29,30}$. However, an emphasis on the importance of advance care plans may create a picture of unhelpful focus on future crises and adverse events ${ }^{31}$, while residents' priorities rest on day-to-day life and being engaged in meaningful relationships within the care home ${ }^{32}$. The participants in this study were aware of their own vulnerability and the uncertainties that they lived with. This contrasts with systems of care that often try to control and ameliorate uncertainty by the use of protocols and in this instance advance care plans ${ }^{33,34}$. Ironically, it was many of the older people's recent and 
everyday experiences that had taught them how uncertain their future was, not least that living in residential care meant there was still the possibility of long term care in other settings. Although, findings for this population, challenge those that suggest that older people as they become more unwell want 'more' intervention not less ${ }^{35}$.

The study cannot claim to be representative of all (residential) care homes in England; the sample selected was fairly typical of those found in the industry and the views held by the residents were similar throughout the six care homes. Care must be taken when extrapolating these findings to the wider population. To differing degrees care staff acted as gate-keepers to residents ${ }^{36}$. This study is limited in that the older people participating may have been more articulate and not in as poor health or cognitively impaired as those who did not participate. The interviews relied on memory and not observation of daily interactions. The complexity of formal advance care plans or nominations of proxy decision-makers is a difficult problem ${ }^{35}$ and this study took place in the early months of the full implementation of the Mental Capacity Act 2005 which has clarified the legal framework ${ }^{37}$. Very few residents seemed to be considering the period of time before death, when they might need more support, comfort, pain-relief or when they would face decisions about stepping up, continuing or having less treatment. Entry to a care home was not of itself a trigger for older people to engage with issues of mortality, limited life expectancy and refusal of treatments.

\section{Conclusion}

This study by focusing on older people who become increasingly frail, because of a steadily diminishing ability to cope with inevitable but unpredictable episodes of ill health, recognises that these older people have particular experiences and expectations about dying. By understanding their expressed needs, experiences and expectations for support and end of life care these findings can inform how primary health and palliative care services introduce, discuss and tailor existing frameworks and programmes of end of life care. 
The importance of relationships between older residents and staff that give purpose and value to the older person's life in a care home is well documented ${ }^{38,39,40}$. Care homes operate in an environment which supports life and death in close quarters ${ }^{41,42}$. The goals of advance care planning are to avoid crisis driven decision making, and unwanted and inappropriate treatments. However, Sudore and Fried ${ }^{43}$ (2010) suggest that there is a need to shift the focus from what they describe as premature decisions based on incomplete information to preparing individuals and their families (and in this instance care home staff) for the types of decisions and conflicts they may encounter when they do have to make in-the-moment decisions. Within an environment where there is continuity of contact, the opportunity to build relationships and conversations over time, discussing and documenting end of life care preferences can and should be threaded through conversations about what is important to older people and their families about living in a care home. In this context, advance care plans that document an ongoing dialogue may be more helpful at the time of decision making. Not least, because as participants in this study identified they are not (and do not always want to be) the only ones in control of their care. Furthermore, understanding why older people may not want to talk about the future and dying may help practitioners and care home staff prioritise and address what is important to the older person and inform how advance care planning is first raised and discussed ${ }^{44}$.

\section{Acknowledgement}

This report outlines independent research commissioned by the National Institute for Health Research (NIHR - PB PG 0906 11387). The views expressed in this report are those of the authors and not necessarily those of the NHS, the NIHR or the Department of Health. 


\section{References}

1. Laing and Buisson. Care of elderly people UK market survey 2009. London. 2009.

2. Office of Fair Trading. Care homes for older people: A market study. London: Office of Fair Trading, 2005.

3. Froggatt $\mathrm{K}$ and Parker D. Care homes and long-term care for people with dementia. In: Hughes J, Lloyd-Williams M and Sachs G (eds.) Supportive care of the person with dementia. Oxford: Oxford University Press. 2010, pp. 181-8.

4. Lynn J. Learning to care for people with chronic illness facing the end of life. JAMA 2000: $284 ; 2508-11$.

5. Michell SL, Teno JM, Kiely DK, Michele LS, Jones RN, Prigerson HG et al. The clinical course of advanced dementia. N Engl J Med 2009; 361(16): 1529-1538.

6. Department of Health. End of Life Care Strategy - Promoting High Quality Care for all Adults at the End of Life. London: Department of Health, 2008.

7. National End of Life Care Programme (NEoLCP) (2010). Supporting people to live and die well: A framework for social care at the end of life: Report of the Social Care Advisory Group of the National End of Life Care Programme. National Health Service. 2010.

8. Larson EB, Shadlen M, Wang L, McCormick WC, Bowen JD, Teri L. et al. Survival after the diagnosis of Alzheimer's disease. Annals Intern Med 2004; 140: 501-509

9. Lynn J. Caring at the end of lives. N Engl J Med 1996; 335: 172-178.

10. Goodman C, Evans C, Wilcock J, Froggatt K, Drennan V, Sampson E. et al. End of life care for community dwelling older people with dementia: an integrated review. Int J of Geriatr Psychiatry 2010; 25: 329-337.

11. Ternestedt BM and Franklin LL. Ways of relating to death: views of older people resident in nursing homes. Int J Palliat Nurs 2006; 12(7): 334-340.

12. Evans C. and Goodman C. Changing practice in dementia care for people in care homes towards the end of life. Dementia 2009: 8(3): 424-431. 
13. Hubbard G, Downs MG. and Tester S. Including older people with dementia in research: Challenges and strategies. Ageing and Mental Health 2003; 7(5): 351- 362.

14. Cantley C, Woodhouse J and Smith M. Listen to Us. Newcastle: Dementia North. 2005.

15. Godwin B and Waters H. 'In solitary confinement': Planning end-of-life well-being with people with advanced dementia, their family and professional carers. Mortality 2009; 14(3): 265-285.

16. Dewing J. From ritual to relationship: A person centred approach to consent in qualitative research with older people who have dementia. Int J Soc Research and Practice 2002: 1(2): 156-171.

17. Dewing J. Participatory research: A method for process consent with persons who have dementia. Int J Soc Research and Practice 2007: 6(1): 11-25.

18. Mental Capacity Act. (Appropriate Body) (England) Regulations 2006. London: The Stationery Office, 2006.

19. Rycroft-Malone J, Kitson A, Harvey G, McCormack B , Seers K, Titchen A et al. Ingredients for change: revisiting a conceptual framework, Quality and Safety in Health Care 2002; 11(2): 174-80.

20. Clarke A and Warren L. Hopes, fears and expectations about the future: what do older people's stories tell us about active ageing? Ageing and Society 2007; 27: 465-488.

21. Seymour J, Witherspoon R, Gott M , Ross, H and Payne S. End of life care. Bristol: Policy Press, 2005.

22. Gott M, Small N, Barnes S, Payne S and Seamark D. Older people's views of good death in heart failure: Implications for palliative care provision. Soc Sci Med 2008; 67: 1113-1121.

23. Lloyd L. Dying in old age: promoting well-being at the end of life, Mortality 2000; 5(2): 171188.

24. Samsi K and Manthorpe J. "I live for today": A qualitative study investigating older people's attitudes to advance planning. Health Soc Care Community 2011; 19(1): 52-59. 
25. Howarth G. 'Just live for today': Living, caring, ageing, and dying'. Ageing and Society 1998; 18: 673-89.

26. Phoenix C, Smith B and Sparkes AC. Experiences and expectations of biographical time among young athletes: A life course perspective. Time and Society 2007; 16(2/3): 247-268.

27. Teno JM and Lyn J. Putting advanced care planning into action. The Journal of Clinical Ethics 1996; 7(3): 205-13

28. Seymour J, Gott M, Bellany G, Clark D and Ahmedzai SH. Planning for the end of life: the views of older people about advance care statements. Soc Sci Med 2004; 59: 57-68.

29. Badger F, Thomas K and Clifford C. Raising standards for elderly people in care homes. Eur J Palliat Care 2007; 14(6): 238-241.

30. Shaw Kl, Clifford C, Thomas K and Meehan H. Improving end-of-life care: a critical review of the Gold Standards Framework in primary care. Palliat Med 2010; 24(3): 317-329.

31. Teno JM. Advance directives for nursing home residents, JAMA 2000; 283(11): 1481-1482.

32. Nolan M, Davies S, Brown J, Wilkinson A Warnes, T, McKee, $\mathrm{K}$ et al. The role of education and training in achieving change in care homes: a literature review. J Res Nurs 2008; 13(5): 411-433.

33. Evans C. The analysis of experiences and representations of older people's health in care homes to develop primary care nursing practice. Unpublished PhD. London: Kings College, 2008.

34. Dartington T. Managing vulnerability: the underlying dynamics of systems of care. London: The Tavistock Clinic Series (Karnac), 2010.

35. Winter $L$ and Parker B. Current health and preferences of life-prolonging treatment: $A n$ application of prospect theory to end-of-life decision-making. Socl Sci Med 2007; 65: 16951707. 
36. Goodman C, Baron N, Machen I, Stevenson E, Evans C, Davies SL et al. Culture, consent, costs and care homes: enabling older people with dementia to participate in research. Ageing and Mental Health 2010a; (In press).

37. Manthorpe J, Heath H, Charles N, Harris J. and Samsi K. 'Early days': Knowledge and use of the Mental Capacity Act 2005 by care home managers and staff. Dementia 2010 (in press).

38. Bern-Klug M. A framework for categorizing social interactions related to end-of-life care in nursing homes. The Gerontologist 2009; 49(4): 495-507.

39. Froggatt K, Davies S. and Meyer J. Research and development in care homes: setting the scene. In: Froggatt K, Davies S and Meyer J (eds) Understanding care homes: $A$ research and development perspective. London: Jessica Kingsley, 2009. pp. 9-22.

40. Brown Wilson C and Davies S. Developing relationships in long term care environments: The contribution of staff. J Clin Nurs 2009; 18: 1746-1755.

41. Froggatt K. Palliative care and nursing homes: where next? Palliat Med 2001; 15: 42-48.

42. Froggatt K. Life and death in English nursing homes: sequestriation or transition? Ageing and Society 2001; 21: 319-332.

43. Sudore RL and Fried TR. Redefining the "planning" in advance care planning: preparing for end-of-life decision making. Annals of Internal Medicine 2010: 153: 256-261

44. Schickedanz AD, Schillinger D, Landefeld C S, Knight SJ, Williams BA and Sudore RL. A Clinical framework for improving the advance care planning process: start with patients' selfidentified barriers. J Am Geriatr Soc 2009; 57(1): 31-39. 
Table 1: Resident Characteristics ( $n=121)$

\begin{tabular}{|l|c|}
\hline Gender & $77.7 \%$ males; 22.3\% females \\
\hline Age Range & $61-101$ years \\
Mean Age & 87.5 years \\
\hline Length of stay in care & 18.5 months (median) \\
home & 26.8 months (mean) \\
\hline Range of Length of Stay & 0.2 months to 276.9 months \\
\hline
\end{tabular}

Table 2: Barthel dependency scores for residents in all care homes at time 1

\begin{tabular}{|l|l|l|l|l|l|l|}
\hline Dependency & High & Medium & Low/Medium & Low & Very Low & Totals \\
(score range) & $(0-4)$ & $(5-8)$ & $(9-12)$ & $(13-16)$ & $(17-20)$ & \\
\hline All care & $5 \%$ & $21 \%$ & $22 \%$ & $30 \%$ & $22 \%$ & $100 \%$ \\
homes & $(n=6)$ & $(n=24)$ & $(n=26)$ & $(n=35)$ & $(n=26)$ & $(n=117)$ \\
\hline
\end{tabular}

